\title{
Exploring Design Principles for Sharing of Personal Informatics Data on Ephemeral Social Media
}

\author{
DANIEL A. EPSTEIN, University of California, Irvine, USA \\ SIYUN JI, University of California, Irvine, USA \\ DANNY BELTRAN, University of California, Irvine, USA \\ GRIFFIN D'HAENENS, University of California, Irvine, USA \\ ZHAOMIN LI, University of California, Irvine, USA \\ TAN ZHOU, University of California, Irvine, USA
}

\begin{abstract}
People often do not receive the engagement or responses they desire when they share on broad social media platforms. Sharers are hesitant to share trivial accomplishments, and the emphasis on data often results posts that audiences find repetitive or unengaging. Ephemeral social media's focus on self-authored content and sharing trivial accomplishments has the potential to ameliorate these challenges. We explore design principles for incorporating personal informatics data like steps, heart rate, or duration in datadriven stickers as a first step towards integrating these data into ephemeral social media. We examine the effect of a sticker's presentation style, domain, domain-relevance, and background through three surveys with 506 total participants. We uncover the importance of domain-relevant backgrounds and stickers, identify the situational value of stickers styled as analogies, embellished, and badges, and demonstrate that data-driven stickers can make ephemeral content more informative and entertaining, discussing implications for platforms and tools.
\end{abstract}

CCS Concepts: • Human-centered computing $\rightarrow$ Human-computer interaction (HCI); User Studies; Social media

\section{KEYWORDS}

Ephemeral social media; Social sharing; Snapchat; Stories; Personal Informatics; Personal Data

\section{ACM Reference format:}

Daniel A. Epstein, Siyun Ji, Danny Beltran, Griffin D’Haenens, Zhaomin Li, Tan Zhou. 2020. Exploring Design Principles for Sharing of Personal Informatics Data on Ephemeral Social Media. In Proceedings of the ACM on Human-Computer Interaction. Vol. 4, No. CSCW2, Article 95, October 2020. 24 pages. https://doi.org/10.1145/3415166

\section{INTRODUCTION}

Apps and devices that track personal informatics data such as physical activity in Fitbit, location in Swarm, or music listening history in Spotify are increasingly allowing people to share that data with others online. Many apps support sharing data with friends or strangers who use the

Permission to make digital or hard copies of all or part of this work for personal or classroom use is granted without fee provided that copies are not made or distributed for profit or commercial advantage and that copies bear this notice and the full citation on the first page. Copyrights for components of this work owned by others than the author(s) must be honored. Abstracting with credit is permitted. To copy otherwise, or republish, to post on servers or to redistribute to lists, requires prior specific permission and/or a fee. Request permissions from Permissions@acm.org.

2573-0142/2020/October - $95 \$ 15.00$

Copyright is held by the owner/author(s). Publication rights licensed to ACM.

https://doi.org/10.1145/3415166

PACM on Human-Computer Interaction, Vol. 4, No. CSCW2, Article 95, Publication date: October 2020. 

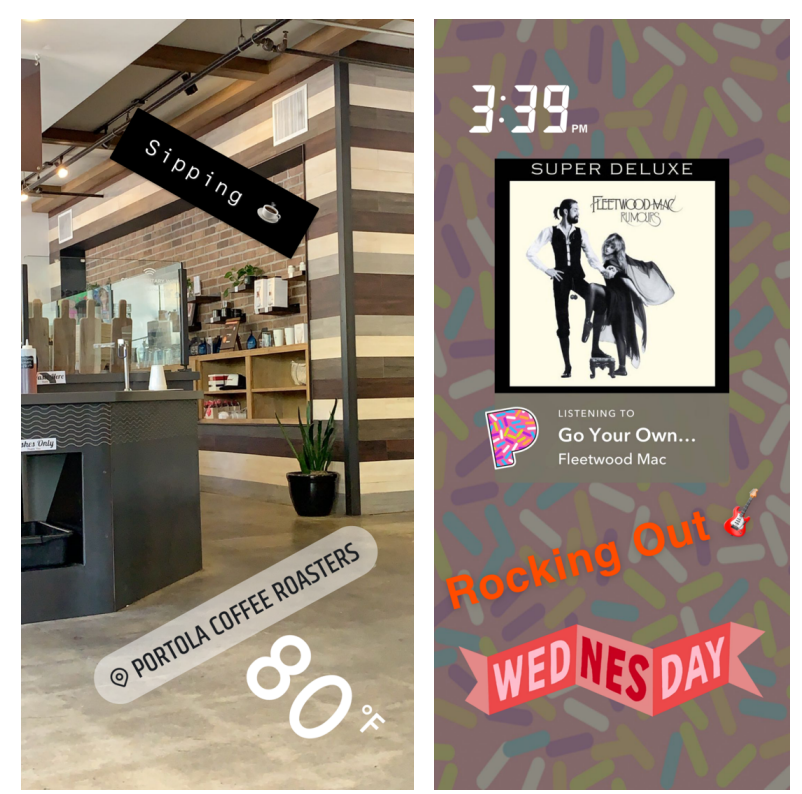

Figure 1. Current ephemeral social media supports adding some experiential data about a person such as time of day, temperature and tagged location, or the music currently being listened to.

same app (e.g., leaderboards on Fitbit, Spotify's friends feed) as well as sharing that data with a broader audience of friends and family who may not have experience tracking the same data (e.g., over a social network like Facebook or Twitter or over SMS $[17,45,46])$. These sharing experiences can support people in being held accountable to their goals [14,56], getting advice $[40,44,54]$, and celebrating their achievements [69] and can support audiences in learning more about the person sharing $[8,36]$ or feeling more connected to them $[5,26]$.

Prior work suggests that sharing personal informatics data over broad-purpose social platforms like Facebook, Twitter, Instagram, and SMS has the potential to help people reach a more diverse audience and communicate aspects of their identity [19,45]. However, in practice, people are often concerned that their activities and accomplishments are too trivial to share to their broad networks on these platforms [18,45,47]. Sharing audiences similarly express limited interest and rarely respond to such content $[19,37]$, often because it appears system-generated rather than authored by the sharer. As a result, people do not receive the responses they seek when they use social platforms to share their self-tracked data [45].

Sharing personal informatics data on ephemeral social media platforms such as Snapchat and the Story features of Instagram and Facebook have the potential to provide the similar benefits to accountability and advice while mitigating some of the concerns people often have. People often use ephemeral platforms to share more mundane life events with closer networks $[9,41,60,66]$, potentially ameliorating sharer's concerns that shared questions or accomplishments are too trivial for the audience. Ephemeral social media emphasizes usergenerated content through annotation of images or videos annotated with text or stickers, further addressing concerns that shared personal informatics data system-generated. Ephemeral platforms often support adding experiential data, such as location, weather, and time of day (Figure 1). Apps like Pandora, Spotify, Netflix, Fitbit, and Strava include features to let people share data they are collecting on ephemeral platforms (e.g., what they are watching or listening to, how far they have walked or run), typically exporting the data as a background image which can be annotated in the ephemeral platform. 
In this work, we examine and extend how current systems support exporting self-tracked data to explore design principles for sharing personal informatics data on ephemeral social media. We specifically explored and evaluated the design of overlays (e.g., stickers) in five commonlytracked and often-shared domains. Our three sticker presentation techniques (badges, embellished, and analogy) draw on techniques to make data interpretable, relatable, and memorable [7,11,29,30]. We conducted three online surveys with 506 total participants to understand how content with personal informatics data's domain, domain-relevance, presentation style, and background effects interest and attitude towards the content. Takeaways from these studies can inform how personal informatics data can be integrated into overlays available on ephemeral platforms. We contribute:

- A design space for how personal informatics data can be incorporated into ephemeral social media. We specifically identify three strategies: stickers with badges, embellishments, and analogies. We explore these design ideas by developing stickers for five domains (steps, music, heart rate, calories, and duration)

- An empirical evaluation of how people's perceptions of overlays vary on four dimensions: presentation style, data domain, relevance of the overlay to that data domain, background. Our findings suggest that: (1) domain-relevant backgrounds and stickers are more useful and entertaining; (2) some data domains detract from the shared content; and (3) all three presentation styles are circumstantially useful.

\section{Background}

This work builds on prior approaches to sharing personal informatics data, studies of ephemeral social media, and techniques for making measurements more interpretable.

\subsection{Sharing Personal Informatics Data}

Personal informatics examines the idea of collecting personally relevant information for later self-reflection or self-knowledge [35]. Although people often collect the data for selfimprovement (e.g., to set a goal, to be aware of their current practices), others self-track for curiosity or to support later reminiscence [20]. There is no agreed-upon definition of what kinds of data can, or cannot support self-knowledge [63]. In practice, personal informatics examines people collecting a variety of data relating to personal wellbeing including physical activity, sleep, and food, as well as non-wellbeing metrics such as productivity, location, and finances.

Prior work has examined social media sharing practices in many domains in which people track data about themselves, including physical activity [15,19,26,45,46,57], biometrics (e.g., heart rate) $[17,37,38]$, food [14,21,39], location [5,6,36], music [53,59], and finances [12]. When people share, they often aim to celebrate achievements (e.g., weight loss) $[31,56]$, demonstrate struggles (e.g., in managing a chronic illness) [54], or curate a certain image of themselves (e.g., as athletic or adventurous) [25]. However, people often fear their accomplishments or struggles are not significant enough to warrant sharing on social media [18,45,47].

Some apps for collecting personal data facilitate reaching people who do not use the app, such as through broad social networking platforms like Facebook and Twitter (e.g., [45,46]) or through direct communication via SMS or Email (e.g., [37,45]). This broader sharing allows people to share with and receive feedback from friends and family, people whose support can be particularly meaningful [47]. However, people tend to receive more support and advice when 
sharing among close ties [5,26,38]. Prior work has suggested that data sharing systems should therefore support configurable and informal groups [22,65].

Sharers of personal informatics data often desire more audience response [19,45]. Many apps automatically push data to social platforms when it is collected (e.g., when food is logged in MyFitnessPal or a run in RunKeeper which audiences often view as impersonal [19,37], especially when it involves canned messages or generic badges. Instead, they prefer when the sharer authors a message or post themselves, such as by explaining the importance of the moment or including a picture [19]. Custom authoring around self-tracked data has begun to be explored systems like DataSelfie, which enables mapping data to visual elements like colors or sizes in images [32].

\subsection{Ephemeral Social Media}

Compared to archival social media (e.g., Facebook and Twitter feeds), ephemeral social media becomes unavailable to the audience after a specified amount of time. In Snapchat, direct messages are typically visible for a few seconds $(<10)$, while network-accessible "Stories" are viewable for 24 hours. Stories often include multiple posts in sequence, enabling someone to share multiple stages of an event or activity as it unfolds. Ephemeral platforms are usually used among smaller, closer networks $[9,60,66]$ to share more mundane, everyday events $[9,41,60,66]$ than archival platforms. People often find activities and accomplishments around tracked data too minor to share to archival social media $[18,45]$ and likely not of interest to the broad audiences there $[19,47]$. Since smaller networks and sharing everyday events are typical on ephemeral platforms, they have the potential to better serve people's goals for sharing selftracked data [22].

Although most ephemeral social media posts emphasize photo- and video-based content $[50,66]$, people often annotate that content with text, filters, or stickers to add context. Annotations are widely used: one study showed $75 \%$ of Snaps sent included text or a drawing alongside media [50]. People use these annotations to clarify meaning $[9,60]$ and better explain their situation [9], similarly to how they use contextual information on other social platforms $[6,28,36]$. Alhabash \& Ma report that self-documentation and self-expression motivate people to use Snapchat more than archival social media platforms like Facebook and Twitter [1]. The annotation features of ephemeral social media help people author stories and posts which express aspects of their personality [2]. Annotations could be effective in helping people author content which feels personal from their self-tracked data.

Today, ephemeral platforms include a few filters and stickers which add personal data. Snapchat, Instagram, and Facebook stories support adding location, weather, and time, like in Figure 1. Other apps like Pandora, Spotify, Netflix, Fitbit, and Strava include features to let people share self-tracked or personal data specific to these apps on ephemeral platforms (e.g., what they are watching or listening to, how far they have walked or run). Like in Figure 1 (right), these apps typically export the data with a background image which can be annotated in the ephemeral platform, rather than the context being annotations. Although this focus on the data itself draws attention to that information, people tend to find seeing pictures alongside self-tracked data more interesting [19].

A few research apps have explored ephemeral messaging of self-tracked data, finding that the shared data can help support connectedness and awareness [5,6,38]. These systems often use abstract representations of the data, such as sending a bouncing ball which reflects a person's 
heart rate [38] or a vibration signal when a person arrives at a tagged location [5]. These abstract representations help facilitate playfulness [38] and peace of mind [5].

\subsection{Making Numeric Data Interpretable and Memorable}

Shared personal informatics data is often numeric in nature (e.g., step counts, heart rate in beats per minute, calories consumed). However this numeric emphasis can come across as impersonal [19], and people often struggle to make sense of raw numbers and measurements [48]. The information visualization field has examined many methods for presenting numbers in broadlyinterpretable ways. A common strategy is re-unitizing the measurement using familiar, realworld objects [13]. Relevant analogies can help people comprehend measurements, such as comparing distances to nearby or well-known landmarks $[3,33]$ and weights or sizes to those of common objects [30]. Another strategy is to remove graphs entirely, instead summarizing the numbers in a natural-language sentence [10].

When visualizing measurements, Tufte argues that chartjunk, or any sort of visual embellishment, makes it more difficult to draw accurate conclusions from the data [58]. Versions of embellishments often occur in graphic design and news media, where graphs are embedded into animals or objects to draw attention [7]. In spite of the potential risks to interpretability, embellishment can be used to drive engagement and interest in data visualization [29]. Embellished visualizations also tend to be more memorable [7,11]. When graphic designers imagined representations of step activity, they used embellishments to quickly convey the data type and highlight accomplishments [3].

A numeric visualization's memorability and attractiveness is often in tension with its interpretability and accuracy [43]. Visualizations designed for scientific communities tend to emphasize utility. But Pousman \& Stasko argue that in casual settings, such as a person sharing their habits on social media, visualizations should aim to be engaging without the same level of regard for utility or interpretability [51].

\section{Design of Personal Data-Driven Overlays}

We explored the design space of displays of personal driven data through studying prior approaches and creating our own, similar to other open-ended design explorations of selftracking systems [24]. We sketched overlay ideas and representative use scenarios on paper, iteratively refining ideas through discussion as a group and informally evaluating by showing our ideas to others. Our initial ideas were motivated by narrative visualizations [52] of one's own data (e.g., year-end Strava and Fitbit reports), animations showing passage of time or training montages (e.g., flipping calendars or climbing up stairs), and public data displays like at sporting events or public art (e.g., noise meters or sand timers). When we reviewed these examples, we considered how they would need to be adapted for the ephemeral medium and how they could be generalized. We arrived at four principles for designing overlays, aiming for overlays to be:

- Playful: to align with platform and casual visualization norms [51], we made our overlays bright and lighthearted. Although traditional charts are common in sharing features [19], we avoided them because we felt people might view them as too serious for the platform. We aimed to align our designs with Snapchat's aesthetic, but kept them general enough to fit other ephemeral platforms (e.g., we did not include Bitmoji or other Snapchat-specific features). 
- Succinct: posts are typically available for 10 seconds or less, so we aimed to use simple shapes and reduce text.

- Relatable: we aimed to develop overlays which used easily-recognizable objects and required minimal location or cultural context. For example, we used everyday objects and well-known animals. Our overlays catered to American audiences in practice, such as comparing duration to the length of a football game and showing calorie information on a fork.

- Broad: we aimed for each overlay to apply to many scenarios. For example, calorie badges initially annotated the calorie count on stickers of food, but pilot participants found it odd to overlay a sticker of a burger on a picture of a person eating Chinese food.

\subsection{Overlay Design Parameters}

With these principles in mind, we refined our overlay concepts. We identified four parameters on which overlays can vary: presentation style, domain, domain-relevance, and background. We then developed overlays which varied on these parameters. Figure 2 shows examples of the overlays we developed with variance on each parameter, while Figure 3 shows two instantiations of one overlay design.

\subsubsection{Presentation Style}

We identify three distinct graphical styles for overlays, each informed by a body of prior literature:

Badge overlays annotate objects with the specific tracked value, for example a shoe or ribbon with "5,793 steps" written on it. Badge overlays draw inspiration from shareable badges in research and commercial tracking apps (e.g., Fitbit [45]) and typical stickers in commercial ephemeral platforms.

Embellished overlays present common objects as charts, picking one dimension to be the axis and shading the object partway according to the tracked value. We aimed for embellishments to be related to either the tracked value or to frequently-measured amounts. For example, we used the tracked data " 11 plays of The Beatles" to shade a pair of headphones or a speedometer about halfway between endpoints of 0 and 20. Embellished overlays are motivated by prior work on chartjunk, which suggests that the technique may make charts more memorable [7,11].

Analogy overlays re-express tracked values as better-known quantities through comparisons. We aimed to use household or well-known objects or animals for our comparisons. For example, one overlay compared a tracked amount of 2 hours, 42 minutes to the length of "Star Wars" (1.3x the value). Another analogy compared the same value (162 minutes) to just under a minute per pound of a stove ( 210 pounds [42]). Analogies draw from prior work suggesting reexpressions and natural-language sentences can help make measurements more understandable $[10,30]$.

\subsubsection{Domain}

People often track and share personal data in a range of domains for varied motivations [19]. We selected domains which can support the varied reasons people share personal informatics data. We specifically designed for five domains: music, steps, calories, duration, and heart rate. These domains help demonstrate how data might be used to celebrate an achievement (e.g., steps), explain more about a moment (e.g., heart rate, duration), or present a desired identity (e.g., music, 


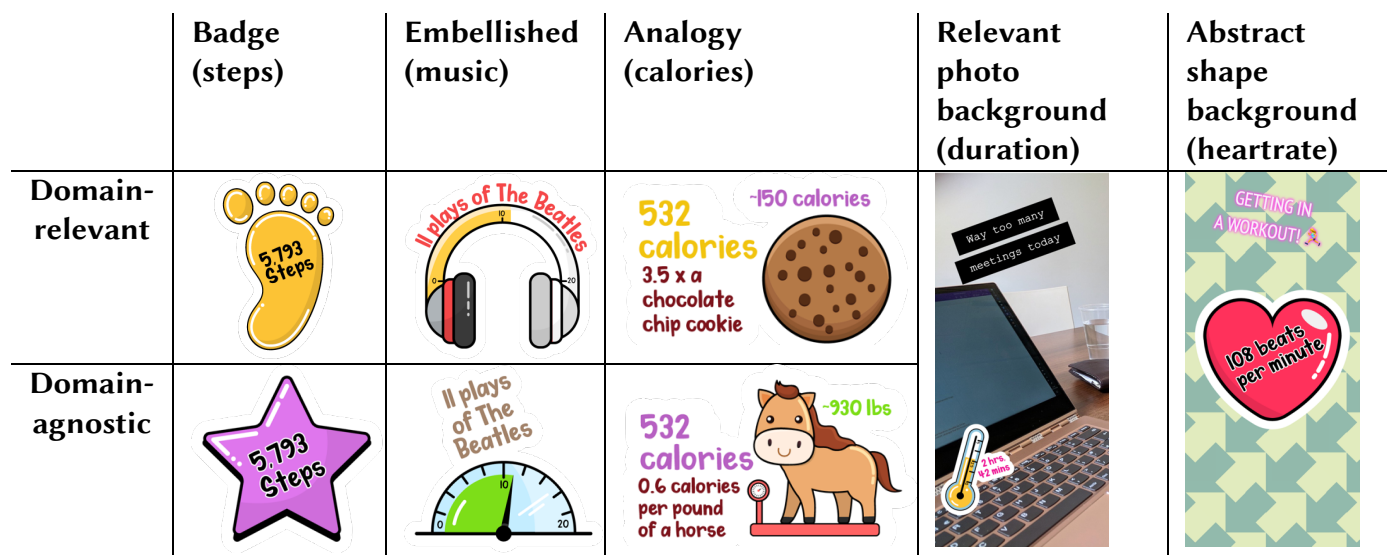

Figure 2. The overlays we created varied on four dimensions: presentation style (badge, embellished, and analogy); domain (music, steps, calories, duration, heart rate), domain-relevance (domain-relevant or domainagnostic), and background (photo and shape).
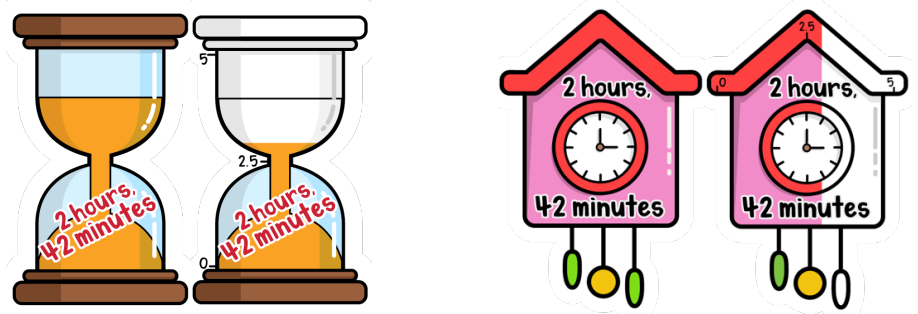

Figure 3. Two domain-relevant concepts for the duration domain, varied for badge (left) and embellished (right) presentation styles.

calories). Each domain refers to a commonly-tracked data unit (e.g., steps for activity, calories for food), though we acknowledge that people may prefer to add other units for the same data type.

\subsubsection{Domain-Relevance}

As demonstrated in previous examples, overlays can use objects or comparisons specifically related to the domain being shared or can use generic values or comparisons. For example, domain-relevant overlays showed music data over headphones or step data over a pair a shoes. Domain-irrelevant overlays used well-known and relatable objects and comparisons, such as a star or a speedometer. However, the object or comparison used in domain-irrelevant overlays is not commonly associated with the domain of focus. Although domain-relevant overlays present a more consistent message across sticker and text, domain-irrelevant overlays support applying to whatever domain is well-suited to a person's sharing goal.

The badge styles of domain-irrelevant overlays used shapes associated with achievement (e.g., a star, badge, or ribbon). Embellished styles used shapes associated with measurement (e.g., a speedometer, beaker, or thermometer). Analogies compared the tracked number to the weight of a common object or animal derived from re-expression aggregators (e.g., 108 BPM is $\sim 1$ beat per pound of an octopus, 11 plays of The Beatles is $\sim 1$ play per pound of a bowling ball) $[30,42,64]$.

\subsubsection{Background}

Most commercial applications which support exporting personal data from their own apps (e.g., Pandora, Fitbit) to ephemeral platforms share the content over a background of abstract shapes 
(Figure 1, right). Alternatively, people tend to use stickers generated natively on ephemeral social media over a relevant photo (Figure 1, left). We made the abstract shape style with public CSS patterns [61], centering the overlay to take up the bulk of the screen (Figure 2). We used Creative Commons photos from Flickr for the relevant photo style, shrinking the overlays and moving them away from the center to avoid hiding the picture. Because most Snaps include text captions [50], we wrote captions to explain the image or data for both background styles.

\section{Evaluation of Data-Driven Overlays}

In ephemeral social media today, overlays can be added to direct messages sent to a specific person (e.g., a Snap) or to messages seen by anyone who chooses to look at the content and has been given access (e.g., an Instagram or Snapchat story). We evaluated our overlays in both scenarios, aiming to answer: How does an overlay's (1) domain and context (e.g., domain, background) and (2) presentation (presentation style, domain-relevance) impact people's perception of shared ephemeral messages and stories? (3) Do people find direct messages or stories with well-regarded overlays more entertaining, informative, or interesting than those without them? Answering these questions will help develop guidelines for ephemeral overlays driven by personal data and determine their utility.

We conducted three studies to answer these research questions. Study 1 examined how the form of overlays effect people's perceptions when directly messaging someone, such as from Snapchat's friend's list. Study 2 examined the effect of the form of overlays in "Story" features where audiences decide to view, such as Instagram or Snapchat stories. Study 3 compared overlays which followed the guidelines from Studies 1 and 2 against a baseline with no sticker. The studies used similar experiment design, response measurements, and recruitment methods. We therefore first describe methods across all three studies, briefly explaining each study's differences in subsequent sections.

\subsection{Experiment Designs}

We used a factorial study design to answer our research questions. We selected this study design to enable varying domain, context, and presentation and evaluating its influence on participant perception and preferences. Participants first consented to participate in the study. They then identified one person who they frequently Snap with to imagine as their conversation partner. They then gave feedback on 6-8 Snaps, answering some demographic questions upon completion.

Stickers and scenarios varied in 120 ways, with each study including a subset of dimensions:

- 5 domains: music, steps, calories, duration, heart rate

- 2 background styles: relevant photo, abstract shapes

- 3 presentation styles: plain, embellished, analogy

- 2 relevance levels: domain-relevant, domain-irrelevant

- 2 message formats: direct messages, stories

All stickers showed the same value for a given domain (e.g., "5,793 steps", "11 plays of the Beatles"). We chose values which felt attainable for most people in their daily lives as to not draw attention to an overly high or low value.

We further sought to control our study for factors which we expected would impact people's perception of Snaps. We aimed to minimize the impact of aesthetic preferences and relatability of Snaps, such as color clash between an overlay and a background or connecting more with an 
analogy to horses than to bears. We therefore generated three overlays for each specific style (e.g., three domain-relevant time embellished, Figure 3). We similarly aimed to minimize the impact of relatability to a particular scenario (e.g., an office worker relating more to a Snap about time in meetings), generating three scenarios for each domain. For example, we wrote captions and found appropriate backgrounds for heart rate Snaps about exercising, running to catch a bus, and giving a presentation. Finally, people tend to be more concerned about selfimage when sending content and more judgmental when they are receiving it $[19,47]$. We therefore sought to control for the impact of role, randomly-assigning participants to be either sharers or recipients for the study. For these reasons, we included 24 control variables:

- 2 roles: sharers, recipients

- 3 overlay instantiations of each domain-presentation-relevance combination

- 3 scenarios/captions

The three studies varied different parameters of the sticker design and scenario space (Table 1). We examine variations in sticker presentation in Study 1 (RQ1-2), narrowing presentation focus in Study 2 but evaluating a new message format (RQ2), and compare message formats in Study 3 using well-received sticker presentation parameters (RQ3). All studies included the same control variables.

\begin{tabular}{l|l|l} 
Study & Independent variables & Message format \\
\hline Study 1 (RQ1-2) & Domain & Direct messages \\
& $\begin{array}{l}\text { Domain-relevance } \\
\text { Presentation style } \\
\text { Background }\end{array}$ & \\
\hline Study 2 (RQ2) & $\begin{array}{l}\text { Domain-relevance } \\
\text { Presentation style }\end{array}$ & Stories \\
\hline Study 3 (RQ3) & $\begin{array}{l}\text { Presentation style } \\
++ \text { "no sticker") } \\
\text { Message format }\end{array}$ & Direct messages \& Stories \\
& &
\end{tabular}

Table 1. The three studies explored different aspects of the overlay design space, together answering our research questions.

To avoid causing confusion by frequently switching study contexts, each participant kept one randomly-assigned domain (in Study 1 only) and role for each of the Snaps (i.e., between subjects). Specific overlay instantiations were randomly assigned per-sticker. Participants saw each independent variable (background style, presentation style, relevance level) and control variable (scenarios/captions) an equal number of times, randomly ordered (i.e., within subjects). The supplemental materials include all stickers, scenarios, and backgrounds used in the studies.

\subsection{Response Measurements}

We use a mix of quantitative and qualitative measures to understand participant opinions on each scenario. Participants rated each scenario on two questions from each of four widely-used scales from online marketing and advertising literature, modified to our domain. Participants also described how they would feel about sending or receiving that snap in an open-ended field. The validated scales measured (1) how entertaining the shared content is (e.g., "I would find this Snap fun to send") [68], (2) attitude toward the content (e.g., "I would feel good about receiving this Snap") [34], and (3) inclination to use the feature (e.g., "I might send this Snap") [34]. For last scale, (4) sharers rated how invasive they would find sharing that content (e.g., "The value I would gain from sending this Snap is worth the information I would give away" ) [67], while recipients rated how informative they found the content (e.g., "I would learn a lot about my close friend or family member from this Snap") [34]. We tailored questions to the message format in 
each study (e.g., Study 2 questions used "Snap Story" versus “Snap”). Participants answered each question on a 7-item Likert scale with endpoints "Strongly Disagree" and "Strongly Agree".

\subsection{Recruitment Methods}

We recruited study participants from Amazon Mechanical Turk. To help ensure response quality and limit spam, we restricted participation to workers with at least $95 \%$ HIT acceptance rate and 1,000 completed HITs. We paid participants $\$ 2.00$ for completing the survey, which took about 10 minutes to complete. We recruited different participants for each of the studies.

We required participants to be at least 18 years old, have a Snapchat account, and average sending or receiving at least one Snap per week. We decided to recruit on Snapchat use specifically because all content on the platform is currently ephemeral, versus other platforms where participants may make less frequent use of ephemeral features or might confuse them with the traditional feeds. It also kept the eligible study population consistent across the three studies.

We recruited 333 respondents for Study 1. We removed 13 participant responses whose selfreported demographics suggested no prior Snapchat use (e.g., 0 weekly Snaps or a fractional Snapchat score), 16 participants who gave every question the same rating on two or more scales, and 41 participants where open-ended responses were nonsensical or unrelated (e.g., "reading about the survey page", "this fell is good and nice") or were identical across many Snaps. We therefore analyzed responses from 263 participants. We recruited 160 participants for Study 2, removing 2 based on demographic responses, 14 for redundant scales, and 21 for unrelated or redundant text. We therefore analyzed 123 responses. We recruited 150 participants for Study 3 , removing 9 based on demographic responses, 9 for scales, and 12 for text, analyzing 120 responses. Table 2 summarizes participant demographics for all three studies. Participants imagined sending Snaps to or receiving Snaps from close friends, family members, or partners. We were

\begin{tabular}{|c|c|c|c|}
\hline Demographic & $\begin{array}{l}\text { Study } 1 \\
\text { ( } 263 \text { people) }\end{array}$ & $\begin{array}{l}\text { Study } 2 \\
\text { (123 people) }\end{array}$ & $\begin{array}{l}\text { Study } 3 \\
(120 \text { people })\end{array}$ \\
\hline Gender & $\begin{array}{l}98 \text { female } \\
162 \text { male } \\
2 \text { non-binary } \\
1 \text { no response }\end{array}$ & $\begin{array}{l}47 \text { female } \\
75 \text { male } \\
1 \text { non-binary }\end{array}$ & $\begin{array}{l}54 \text { female } \\
65 \text { male } \\
1 \text { non-binary }\end{array}$ \\
\hline Age & $\begin{array}{l}\text { Average } 32.0 \\
\text { Min } 19 \\
\text { Max } 75\end{array}$ & $\begin{array}{l}\text { Average } 31.6 \\
\text { Min } 18 \\
\text { Max } 59\end{array}$ & $\begin{array}{l}\text { Average } 32.9 \\
\text { Min } 19 \\
\text { Max } 56\end{array}$ \\
\hline $\begin{array}{l}\text { How many months used } \\
\text { Snapchat }\end{array}$ & $\begin{array}{l}\text { Average } 25.4 \\
\text { Min } 1 \\
\text { Max } 90\end{array}$ & $\begin{array}{l}\text { Average } 24.5 \\
\text { Min } 1 \\
\text { Max } 72 \\
\end{array}$ & $\begin{array}{l}\text { Average } 29.4 \\
\text { Min } 1 \\
\text { Max } 90\end{array}$ \\
\hline $\begin{array}{l}\text { Average number of Snaps } \\
\text { sent/received per week }\end{array}$ & $\begin{array}{l}\text { Average } 33.8 \\
\text { Min } 1 \\
\text { Max } 350\end{array}$ & $\begin{array}{l}\text { Average } 46.0 \\
\text { Min } 1 \\
\operatorname{Max} 1,000\end{array}$ & $\begin{array}{l}\text { Average } 47.7 \\
\text { Min } 2 \\
\operatorname{Max} 1,000\end{array}$ \\
\hline Snapchat score & $\begin{array}{l}\text { Average } 12,127 \\
\text { Median } 2,332 \\
\text { Min } 5 \\
\text { Max } 312,784\end{array}$ & $\begin{array}{l}\text { Average } 15,591 \\
\text { Median } 2,233 \\
\text { Min } 1 \\
\operatorname{Max} 474,102\end{array}$ & $\begin{array}{l}\text { Average } 18,024 \\
\text { Median } 4,212 \\
\text { Min } 3 \\
\text { Max } 157,505\end{array}$ \\
\hline Number of Snapchat friends & $\begin{array}{l}\text { Average } 10.8 \\
\text { Median } 5 \\
\text { Min } 1 \\
\text { Max } 325\end{array}$ & $\begin{array}{l}\text { Average } 13.4 \\
\text { Median } 4 \\
\text { Min } 1 \\
\text { Max } 500\end{array}$ & $\begin{array}{l}\text { Average } 21.1 \\
\text { Median } 5 \\
\text { Min } 1 \\
\text { Max } 1000\end{array}$ \\
\hline $\begin{array}{l}\text { Imagined sharer or recipient } \\
\text { in study }\end{array}$ & $\begin{array}{l}42 \text { partner } \\
57 \text { family } \\
79 \text { close friend } \\
58 \text { other friend } \\
27 \text { other/unsure }\end{array}$ & $\begin{array}{l}24 \text { partner } \\
17 \text { family } \\
36 \text { close friend } \\
28 \text { other friend } \\
18 \text { other/unsure }\end{array}$ & $\begin{array}{l}15 \text { partner } \\
27 \text { family } \\
39 \text { close friend } \\
21 \text { other friend } \\
18 \text { other/unsure }\end{array}$ \\
\hline
\end{tabular}

Table 2. We surveyed 513 Snapchat users recruited from Amazon Mechanical Turk across three studies. 
occasionally unsure of the relationship when participants gave names (e.g., "Mike", "Corrine").

\subsection{Analysis}

We used mixed-effect ordinal logistic models in analysis, treating the Likert data as an ordinal response and taking the median response among the two questions for each scale. We opted for an ordinal response to allow for differences in intervals between successive values, but in testing found few differing significant results between ordinal and linear response models. We treated participant id as a random effect to help account for individual differences in use of or thoughts on Snapchat. We treated each of the varied parameters in our factorial study design as fixed effects. We included interaction effects between domain, presentation, and relevance, as the interaction of these factors varied the actual image of the overlays varied. For example, a domain-relevant badge might be a heart for the heart domain, but a clock for the time domain. Similarly, we presented that same clock as an embellished sticker (Figure 3). We also included a term for how many Snaps they had seen prior. We corrected for multiple comparisons in posthoc tests used with Tukey corrections in pairwise tests and false discovery rate corrections in family-wise tests. We display confidence intervals for each response, and all displayed confidence intervals represent differences in ratings on a 7-point Likert scale.

We removed control terms in each study which had no significant effect for any of the scales to produce simpler models. The overlay instantiation had no measurable effect on any of the scales in any of the studies $(p>0.05)$. The specific scenario and caption tended to impact participant opinions in Studies 1 and 3 (e.g., $p<0.001$ for inclination to use and attitude towards the content), but not in Study 2 ( $p>0.05$ for all scales), suggesting that adding tracked data may be more applicable to some scenarios than others. In Studies 1 and 3, participants assigned to the recipient role were more likely to respond to a Snap more than participants assigned to the sharer role were to share $(Z=3.24, p<0.01,95 \%$ CI 0.28-1.12 higher; similar effect size in Study 3). This aligns with prior findings that people fear oversharing to their audiences [45,47]. In each study, participant's attitude toward the shared content lowered slightly as they saw more Snaps (in Study 1, Z =-3.00, $p<0.01,95 \%$ CI 0.03-0.13 lower per Snap seen; similar effect sizes in the other studies), suggesting fatigue when receiving many Snaps with shared data similar to on other platforms [19].

To analyze participant's open-ended responses to seeing each Snap, we used the quantitative results to help develop our codebook for deductive coding. We developed 12 codes for the first study, 17 for the second, 7 for the third (36 total). Codes included participants finding the sticker relatable, appreciating the presence of a background photo, and expressing a privacy concern with sending the Snap. Two researchers coded a random $10 \%$ of the open-ended responses for each study, finding agreement on most codes ( $\mathbf{\kappa} 0.26-1$, with $35 / 36$ above 0.5 and 28/36 above 0.8 ) and resolving disagreements though discussion. One of the researchers then coded the remaining responses. We labeled at least one code in about half of participant responses: $49 \%$ in Study 1, 53\% in Study 2, 42\% in Study 3.

\subsection{Limitations}

By recruiting on Mechanical Turk, our participant demographics differed from platforms with ephemeral features such as Snapchat, Instagram Stories, and Facebook Stories. Relative to Snapchat and Instagram as a whole, our participants skewed male and older [49,62]. Although our participant's age distribution were closer to Facebook's demographics (e.g., high rates of use 
for people around and over 30) [49], reports suggest younger people tend to create and view stories more often [55]. Participants' Snapchat score was also somewhat lower than past studies [27].

We are unsure whether these demographic differences impacted our participant's perceptions positively or negatively. Our participants' lower frequency of use may have made them less amenable to the playful nature of adding stickers to Snaps (e.g., s47, "thankfully I have no friends that send snaps like the ones viewed in this study"). However, the older demographic may have more purpose-driven uses of the platform where additional data from the sharer could be helpful (e.g., p97, "I learned about [Snapchat] through my sons so they use it a lot more than I do but I use it to keep in touch with them."). Further work should particularly examine sticker opinions of minors, who comprise much of Snapchat's demographic [62].

Although most participants focused their feedback on the design of the overlays, some did not resonate with the content they were asked to imagine sharing or receiving. For example, participants pointed out when the data in the Snaps sounded unlike them when sharing, " $I$ would feel like a poser because I do not listen to the Beatles" (p179) or unlike their social connection when receiving, "My sister is not into Thai food. There is more to this snap taking place" (p68).

We did not ask participants whether they had previously tracked or shared data. Some participants felt that sharing tracked data on social media did not align with their goals or ideals, aligning with prior work [19,37]. For example, some participants mentioned that they would not track the metric, "I would not share it. I don't care about calories" (p92) or that they would not present themselves online in that way, "I don't like to show anxiety in my Snaps so I would feel embarrassed" (p67). However, most participants gave feedback on the design of the overlays, suggesting most were able to imagine what it would be like to share or receive posts with self-tracked data. Deploying a fully realized system with people interested in collecting and sharing data about themselves could help answer whether including data in ephemeral posts supports people's sharing goals and how their friends and family feel about receiving them.

People often record and send videos as backgrounds on ephemeral social media in addition to static photos. Stickers are also often animated. As a first examination of people's perceptions of data-driven stickers, we opted to study only static background photos or patterns and static stickers.

\section{Study 1 Results: Direct Messages}

We focus on how the four dimensions impacted participant perspectives, quoting participant responses with $\mathrm{pXX}$.

\subsection{Presentation Style}

Across domains, participants rated analogies more informative than plain or embellished stickers ( $Z=3.19, p<0.0195 \%$ CI 0.06-0.44 higher). Participants appreciated the information, insight, and fun which analogy stickers provided. 11 participants liked how analogies helped them contextualize the amount, including p66: "the information in the sticker is really interesting; really puts that distance in perspective". p114 and 5 other participants learned something from the comparison: "I also just learned that an average NFL game is 3 hours and 12 minutes long, so I learned a fact at the same time." 11 participants mentioned finding the comparison fun, even when unrelated "I like how it gives a random item as a comparison. It could lead to some funny snaps" (p100). However, 


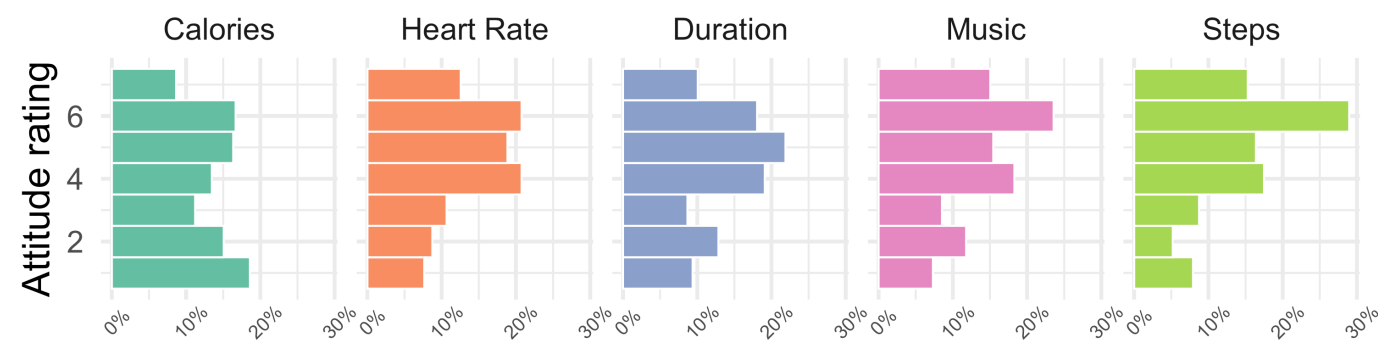

Figure 4. Participants had the highest impression of Snaps of step content and the lowest of Snaps showing calories.

5 participants mentioned that analogy stickers as felt too verbose or complex for Snapchat, such as p128: "it's a little busy and the sticker is kinda small and hard to read."

Comparatively, participants felt badge and embellished stickers were less interesting. 50 and 40 participants used phrases like "a little boring and generic" (p71) or "pretty bland" (p44), to describe badge and embellished stickers, versus 16 participants for analogy stickers. However, participants found badge and embellished stickers useful for demonstrating progress "the sticker itself is honestly very motivating because of the way that it displays progress" ( $\mathrm{p} 121,10$ had a similar sentiment) and easy to interpret "I like the design of this snap because it is not only easy to read but the sticker relate to the beats per minute of the heart. It gets the point across." (p111, 5 others agreed).

\subsection{Domain}

Participants rated adding step data to Snaps slightly higher than other domains, rating their attitude towards the content more highly $(Z=2.54, p<0.0595 \%$ CI $0.01-1.00$ higher; Figure 4$)$ and rating the content marginally more entertaining $(Z=2.47, p=0.07)$. Participants appreciated how steps would help them share accomplishments. 3 participants in the audience role would "cheer them on" (p258), and 3 found the content motivational for themselves "I need to get out and hike" (p256). 4 participants appreciated how the self-tracked data could help a person present their goals, such as p66: "Looks like they're going for 10 thousand steps."

Participants particularly rated analogies to step data more entertaining $(Z=3.95, p<0.00195 \% \mathrm{CI}$ 0.21-0.85 higher) with a higher intention to use $(Z=2.68, p<0.05,95 \%$ CI $0.04-0.68$ higher $)$ and marginally higher attitude towards the content $(Z=2.16, p=0.09)$ than the other presentation styles. p248 felt analogies were particularly well-suited to sharing their accomplishments: "I like that my steps are compared to the Empire State building, it really shows that I have motivation."

Participants rated Snaps with calorie data worse than other domains, feeling less likely to want to send or receive those Snaps $(Z=-2.99, p<0.05,95 \%$ CI $0.09-1.20$ lower), having lower attitude towards the content $(Z=-3.60, p<0.01,95 \%$ CI $0.21-1.29$ lower; Figure 4$)$, and finding the Snaps marginally less entertaining $(Z=2.09, p=0.09,95 \%$ CI 0.10 higher- 0.94 lower). 10 participants felt including the calories detracted from the enjoyment of sharing or receiving food photos, such as p34: "no reason to focus on calories when there's good food like that in front of me" and p125 "I would focus on the food and where they are, rather than the sticker." Participants also felt sharing calories was more invasive than other domains $(Z=3.24, p<0.01,95 \%$ CI $0.22-1.98$ higher). p107 was "worried that it could come across as shaming other's food choices" (7 others expressed a similar sentiment). 
Although participants primarily appreciated when data could be used to explain an accomplishment, a few participants valued how heart rate, duration, or music could be used to make content more relatable. p71 stated, "it's very relatable in the aspect about giving a speech in front of a crowd. [The sticker] gives more tangibility to the senders current state of emotion" (9 others expressed a similar sentiment). 4 participants commented on how the data in these domains was helpful for self-presentation, including p204 "I'd feel proud of my music tastes and happy to share."

\subsection{Domain-Relevance}

We found no significant differences in how participants rated the domain-relevance of stickers ( $p>0.05$ for all scales). On seeing both styles, participants generally appreciated the relatability of domain-relevant stickers. Of a domain-relevant sticker, p111 said "the sticker relates to the beats per minute of the heart. It gets the point across" (8 others expressed a similar sentiment). 21 participants similarly found the domain-irrelevant stickers distracting "I would kind of question why the beaker is relevant because it doesn't seem to relate to exercise or physical activity" (p41) or confusing "I don't think it makes much sense? Why does it say 1 play per pound of an octopus?' (p150).

Some participants appreciated the playfulness of the domain-irrelevant stickers. 6 participants found domain-irrelevant analogies fun, such as p270: "this is a funny and rather amusing snap. The fact that it uses such an absurd comparison is appealing to me." Domain-irrelevant embellished stickers felt atypical to participants: "I really like the beaker filled with some liquid. It looks very cool and out of the ordinary. I would definitely consider using this sticker" (p134, 4 others agreed), while the icons made badge stickers feel celebratory "showing my friends that I am feeling positive about the intense workload by sending a shiny star makes their days go by faster." (p192, 9 others agreed).

\subsection{Background}

Participants rated Snaps with relevant background photos higher than Snaps with abstract shapes on all scales, finding them more entertaining ( $Z=13.39, p<0.001,95 \%$ CI 1.08-1.45 higher) and informative ( $Z=577.18, p<0.001,95 \%$ CI 1.36-1.37 higher) with a higher attitude towards the content $(Z=13.42, p<0.001,95 \%$ CI $1.09-1.46$ higher $)$ and higher intention to use $(Z=12.94$, $p<0.001,95 \%$ CI 1.05-1.43 higher, Figure 5). 35 participants appreciated the background photo when it was included, and 47 participants mentioned disliking the absence of a background photo. 23 participants felt the Snaps were not as interesting when photos were not included, such as p23: "boring. Why is this one so boring. If there's food involved show it off and let me see it. Otherwise keep it to yourself." p106 suggested that she would use a different platform for

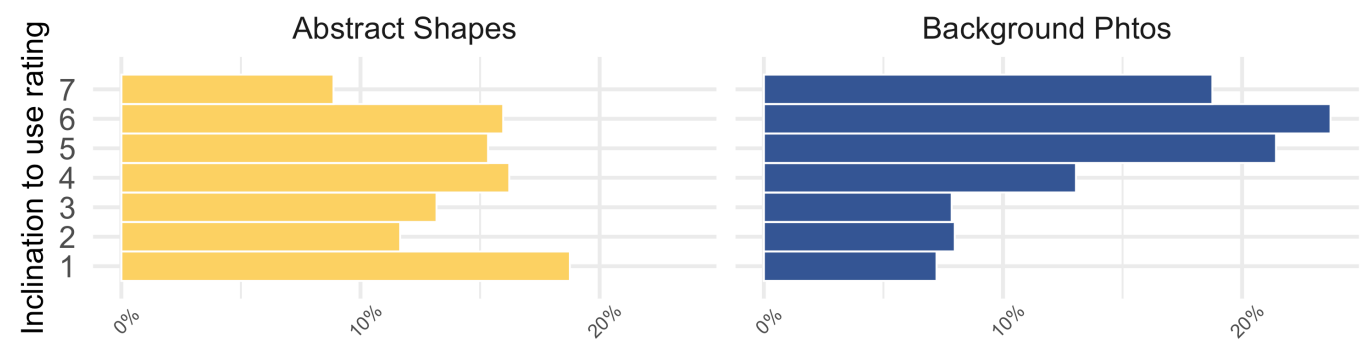

Figure 5. Participants felt they would be less likely to send or receive Snaps with abstract backgrounds than those with photos. 
sharing content without photos: "I wouldn't send this one. I like to use stickers in my snaps, but I'd just send a text if I wasn't going to take a photo." p9 and 4 other participants felt the background provided necessary proof: "I think it is odd to not even have a picture of anything in the snap. I would think people may not believe me."

Sharers also rated Snaps over relevant background photos as more worth the invasion of privacy than Snaps over abstract shapes $(Z=-6.07, p<0.001,95 \%$ CI $0.57-1.12$ higher). We suspect participants felt the utility of including a background photo outweighed potential risks. 22 participants discussed the data domain's invasiveness, but none indicated the presence of a photo increased (or decreased) the concern.

\section{Study 2 Results: "Story" Features}

Participant responses in Study 1 emphasized the importance of including relevant background photos and the need to avoid sensitive domains in direct message Snaps. We therefore selected one domain (steps) and one background (relevant background photos) to examine story features and further evaluate sticker design parameters. We used the same presentation styles (plain, analogy, and embellished) and domain-relevance levels (relevant and irrelevant). The factorial design in Study 2 therefore only had these 6 experimental dimensions (3 presentation styles $\mathrm{x} 2$ relevance levels). Participants saw one Snap of each dimension.

Participants rated 6 stories according to the same scales used in the first study. We drew inspiration from how Instagram presents stories, presenting each image for 5 seconds before transitioning to the next. Our stories consisted of three different photos showing increasing step counts over the course of two hours (Figure 6). Because we wanted to evaluate participants' reaction to the sticker, we kept the sticker's presentation style constant across the three images while varying the amount and any sticker-specific information (e.g., progress on the embellished stickers or the specific numeric analogy). In practice, people may vary stickers according to what best fits with their circumstances.
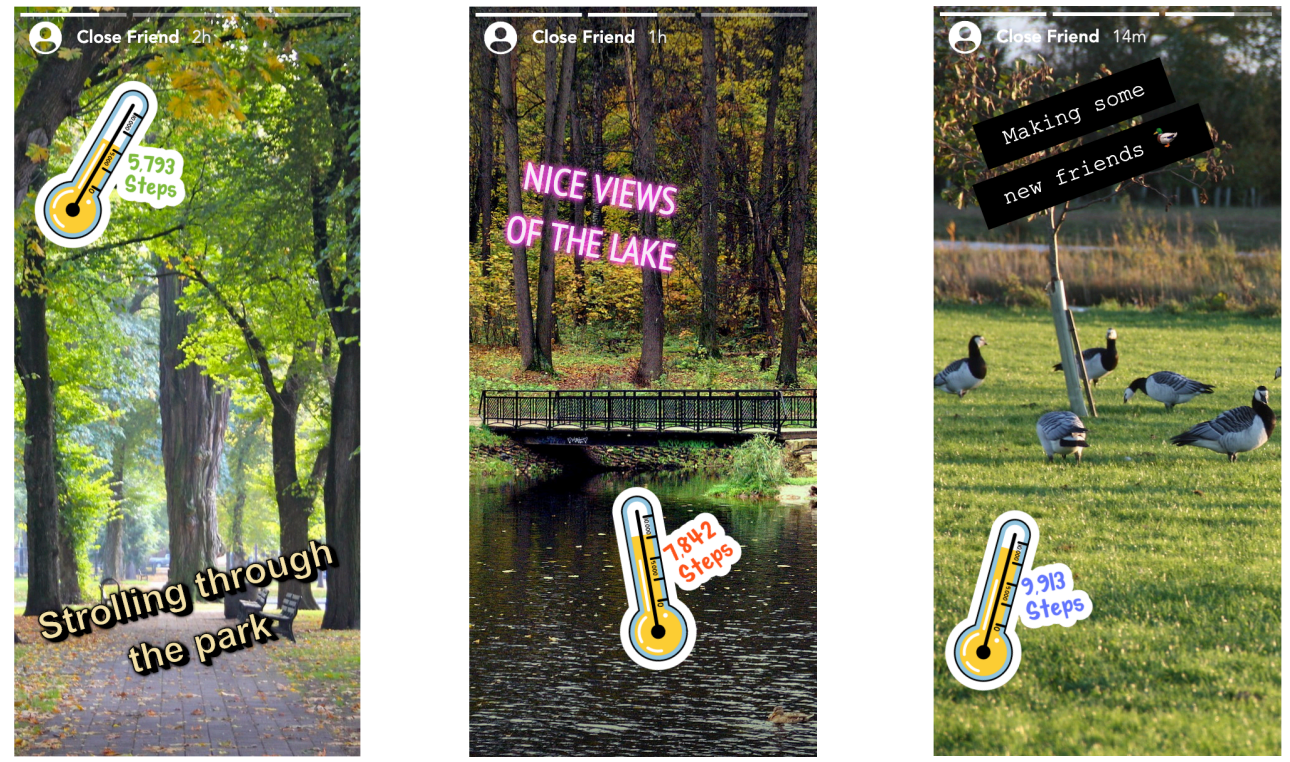

Figure 6. In our study of story features, we varied stickers to show how a person's steps progressed over a two-hour walk. 


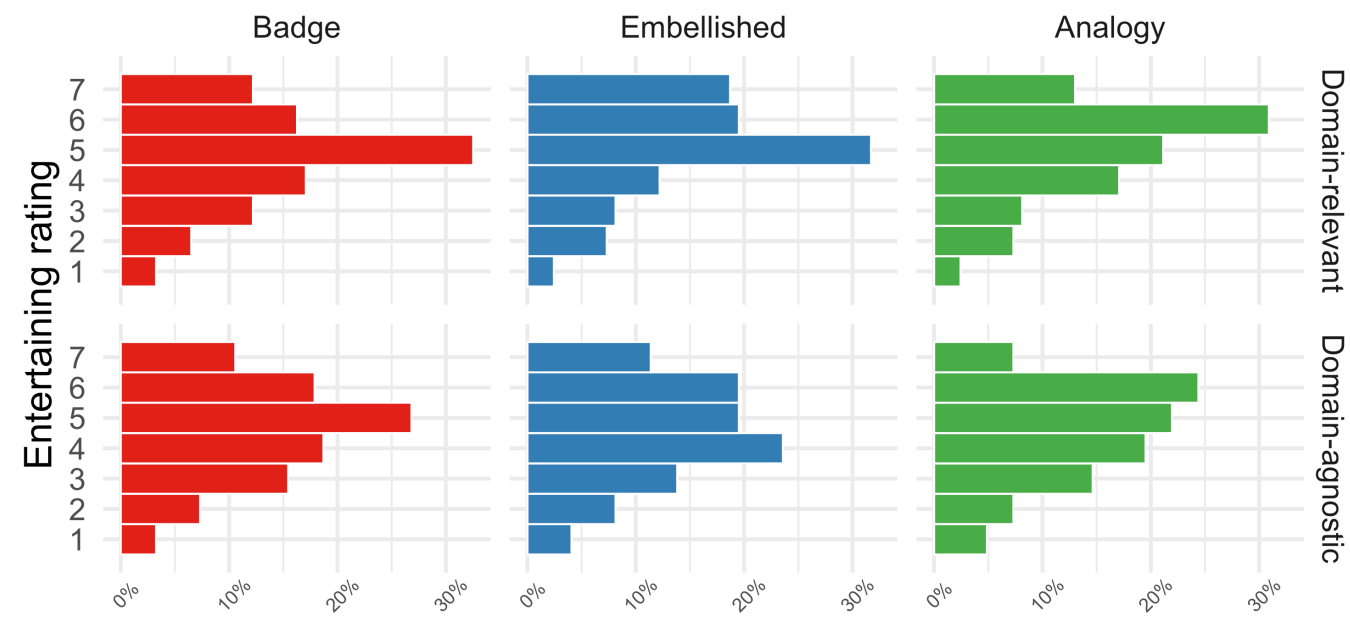

Figure 7. Participants found domain-relevant story Snaps more entertaining than domain-irrelevant ones.

Figure 7 shows participant entertaining ratings across the 6 experimental dimensions. We quote participants with sXX.

\subsection{Presentation Style}

We found no significant differences in how participants rated the three presentation styles ( $p>0.05$ for all scales). 32 participants described similar relatability benefits to analogy overlays as Study 1 participants did, including s114: "This is the best one so far. I like that the stickers give you something to compare to what the steps represent". However, some participants felt badge and embellished stickers were better suited to demonstrating how their step count had progressed over the course of the Snaps in the story. Of an embellished sticker, s37 stated, "I actually kind of like seeing the progression on the sticker and I feel good for my friend" (25 other participants expressed a similar sentiment). s91 felt the bolder, less verbose stickers were a better fit for the divided attention people often give to stories "this one is better.

At least the sticker is less cluttered and the number of steps is made obvious" (9 other participants expressed a similar sentiment). 3 participants felt analogy stickers were too hard to parse, such as $\mathrm{s} 37$ "I still think it requires more thinking than should be needed for a few seconds."

\subsection{Domain-Relevance}

Participants rated domain-relevant story Snaps more entertaining $(Z=2.89, p<0.0195 \%$ CI 0.22 1.13 higher) with a higher attitude $(Z=2.13, p<0.0595 \%$ CI $0.04-0.95$ higher $)$ and higher intention to use $(Z=2.38, p<0.05,95 \%$ CI $0.10-1.01$ higher) than domain-irrelevant Snaps. We found no significant interaction effects between domain-relevance and presentation style $(p>0.05$ on all scales).

While Study 1 participants sometimes viewed domain-irrelevant stickers as playful, the repeated exposure in stories led Study 2 participants to grow tired of the playful elements. s86 said, " $I$ don't really like this sticker. It's a temperature gage which has nothing to do with my progress" (24 participants described a similar sentiment). 7 participants compared how relevant the different types of stickers felt. s93 stated, "I don't really see the correlation between a thermometer and the amount of progress I've made towards a step count goal. The previous stickers used (the shoe, or even the star) made more sense to me. Using a thermometer is a bit reaching." 


\section{Study 3 Results: Overall Interest in Stickers}

We sought to evaluate whether Snaps with overlays driven by personal informatics data provided context and entertainment over Snaps without them, comparing the overlays participants found most entertaining and useful in Studies 1 and 2 against a baseline of Snaps without stickers. Prior work has suggested that many people feel sharing tracked data in social media posts can feel impersonal and elicit less response [19,37], so we chose a baseline of no stickers to evaluate whether our best-received stickers provide enough value to be worth incorporating into future systems. If participants find value in stickers, further work could compare whether the visual medium of stickers add additional benefit over text descriptions of the same content.

All stickers were domain-relevant step overlays with a relevant background photo. We opted to include all presentation styles (analogy, embellished, and badge) as Studies 1 and 2 suggested that use case could influence style utility. Study 3 participants therefore saw 8 Snaps: 4 presentation styles (badge, embellished, analogy, no sticker) x 2 message formats (direct message, story). We quote participants with cXX.

\subsection{Presentation Style}

Participants tended to think sending or receiving data-driven stickers in Snaps would be valuable. Participants found Snaps which contained a sticker to be more informative $(Z=3.70$, $p<0.001,95 \%$ CI $0.06-0.57$ higher) and entertaining $(Z=3.11, p<0.001,95 \%$ CI $0.07-0.56$ higher, Figure 8) than those without. On seeing a Snap without a sticker, 6 participants explicitly commented that they wished they had the context. c68 said, "I prefer the snaps that show the persons step count. they are informative and cooler to see than just a bunch of random pictures", and c1 agreed, adding "this seems very boring now without the sticker?' 5 participants mentioned preferring Snaps without stickers. c49 said: "I love this one without the distracting foot >_>", while c35 felt that the sticker was "cute but I'd rather focus on hiking". 38 participants described Snaps sent without a sticker as boring or uninteresting, including c115 "This just feels boring and might as well be a Facebook post." Comparatively, 20 participants described a Snap with a sticker as boring.

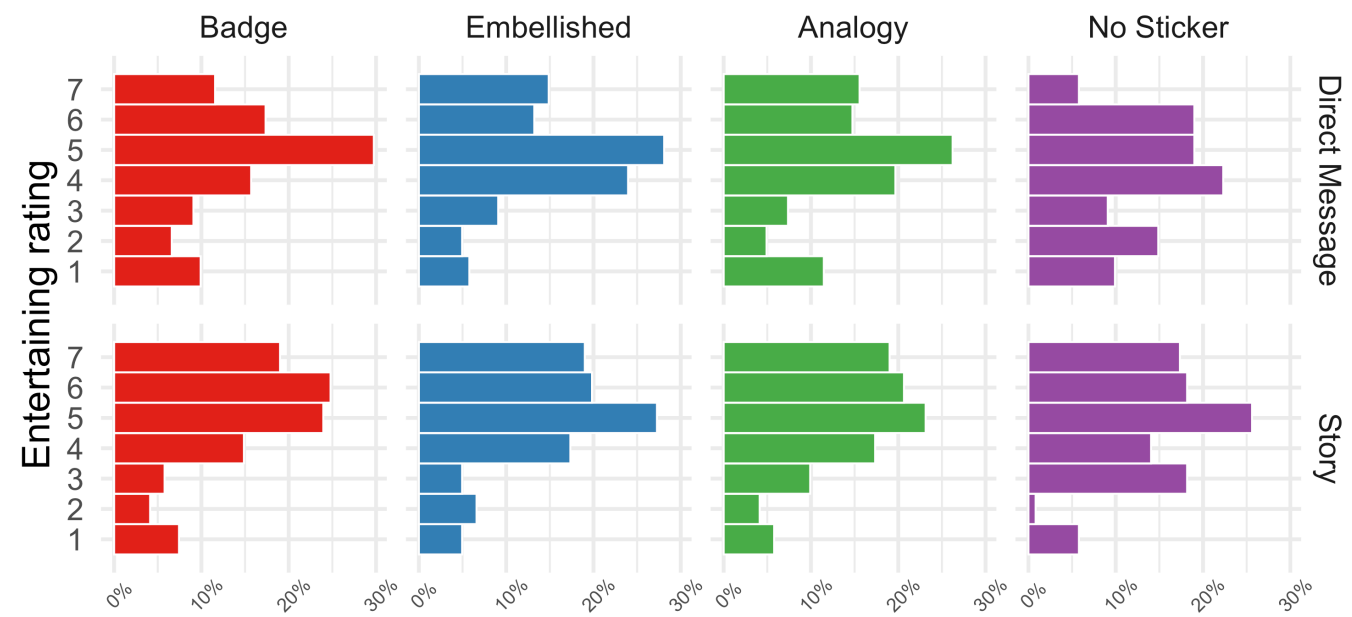

Figure 8. Participants found Snaps with stickers more entertaining than Snaps without and preferred stories over direct messages. 
Although participants found Snaps containing stickers interesting, we did not observe an impact of the presence of a sticker on participant's attitude towards the content $(p>0.05)$ or willingness to send or respond to it $(p>0.05)$. Photos and captions tended to capture attention. 56 participants commented on the Snap's scenery without mentioning the tracked data. For example, when responding to the story in Figure 6, c59 commented "love the lake picture and the picture of your new friends! Did you give them any names?'

Other participants felt that including activity detracted from the quality of the Snap. 13 participants felt that the inclusion of a sticker drew too much attention to their activity, such as c50: "I would feel like I'm too proud of my fitness activity. The sticker looks like it is trying too hard to brag about the distance I've traveled." c25 agreed, adding that the sticker detracted from the attractiveness of the photos he was sharing: "I would be more inclined to send the snap to show the trail and the beauty of nature. I would not put a bunch of stickers bragging about how many steps I took."

We observed no significant difference in preference among the three sticker types $(p>0.05$ for all scales), similar to in Studies 1 and 2. Participants expressed similarly varied presentation style preferences as in the other studies. 8 participants appreciated the simplicity of badge stickers, such as c1, "Yeah, I like the simple stickers much better," but 20 found them plain: "I dont really like the foot sticker, not that interesting" (c116). 13 participants appreciated how the embellished stickers demonstrated progress towards a goal, such as c45 "I like that this sticker tracks my step progress so it would be a fun way to be held accountable for walking more.", but 3 participants found the indication of a goal off-putting "the running shoe is cute, but I don't really like how it measures how close to a goal I am" (c42). 16 participants felt analogy stickers helped them contextualize the distance walked: "yeah, this little golden gate bridge one is pretty cool! It kind of puts things into perspective, you know?" (c102), while 7 found them distracting, such as c27: "It's a very interesting sticker with a comparison of my steps to the golden gate bridge, but I think that it distracts from my actual step count, so I don't think I'd use it."

\subsection{Message Format}

Participants were more inclined to send or respond to Snaps sent via stories than direct messages, rating them more entertaining $(Z=5.81, p<0.001$, 95\% CI 0.45-0.91 higher, Figure 8), with a more positive attitude towards the content $(Z=3.58, p<0.001,95 \%$ CI $0.19-0.65$ higher), and more likely to use it $(Z=4.42, p<0.001,95 \%$ CI $0.29-0.75$ higher). When stickers were not present, participants liked seeing and sharing a day's activities (e.g., "I really like that it is a progression of pictures of their time and that would make me feel good... I would want to know where they are." c120, 15 others expressed a similar sentiment). When a sticker was shown, 13 participants appreciated seeing how step activity progressed over the story, including p104: "this would make it exciting to send updates and continue walking just to see the difference."

We observed no significant interaction effects between presentation style and message format ( $p>0.05$ for all scales), meaning we observed no difference in whether some stickers were more or less applicable to direct messages or stories. Although embellished stickers showed progress between Snaps, people felt plain and analogy stickers also effectively showed how a person's activity had changed. c104 said, "I like how it compares to the Empire State Building so this would make it exciting to send updates and continue walking just to see the difference. This one is so far the best and most exciting one" (13 others expressed a similar sentiment). 


\section{Discussion}

Our studies suggest the potential for ephemeral social media to ameliorate some of the challenges people face when sharing personal informatics data. Although overlays did not impact participant's attitude towards shared content or willingness to share or respond to it, they found Snaps which included overlays more informative and more entertaining. Table 3 summarizes our findings with regards to our research questions.

\begin{tabular}{l|l} 
Research Question & Main Takeaway \\
\hline $\begin{array}{l}\text { RQ1: impact of an overlay's } \\
\text { domain and context }\end{array}$ & $\begin{array}{l}\text { Participants found domains most useful when they helped demonstrate progress toward a goal or } \\
\text { improved relatability. Sensitive domains can detract from the photo being sent. Participants } \\
\text { regarded Snaps where the data is the main shared content as boring. Putting data in sticker-like } \\
\text { overlays can be effective. }\end{array}$ \\
\hline $\begin{array}{l}\text { RQ2: impact of an overlay's } \\
\text { presentation style }\end{array}$ & $\begin{array}{l}\text { Domain-relevant Snaps facilitate more interest and engagement, but domain-irrelevant Snaps can } \\
\text { still add benefit and be entertaining. Analogies are effective for interpreting progress, while } \\
\text { embellishments and badges are useful for celebrating accomplishments. Platforms should likely } \\
\text { support a combination. }\end{array}$ \\
\hline RQ3: whether well-regarded & $\begin{array}{l}\text { Data-driven stickers can add information and entertainment, but tend not to influence people's } \\
\text { decision to share or respond. Further work could compare whether data-driven stickers add } \\
\text { additional benefit over text descriptions of the same data. }\end{array}$
\end{tabular}

Table 3. Takeaways from our three studies.

Participants particularly appreciated when the data domain allowed the sharer to demonstrate progress towards a goal, such as exercise throughout the day. This can be particularly effective in stories, where repeated stickers could show progress over time. Others appreciated how certain data domains made information more relatable or present a certain image of themselves. However, as echoed in prior work, the inclusion of data can be unwelcome in some sensitive domains like calories for food [16] or can come across as bragging about an accomplishment, such as in physical activity [19].

Our findings suggest that overlays are better-received when their visuals are relevant to the domain being shared and when they are used in conjunction with relevant background photos. Practically, a tool may be unable to develop domain-specific overlays which align with the plethora of domains people track about themselves which could add valuable context. Domainirrelevant overlays such as stars or ribbons, or even odd but fun comparisons such as comparing the number to a weight, may still help a person add context in new domains. However, our findings align with prior work on non-ephemeral platforms indicating that people tend not to find sharing tracked data interesting on its own [19,37,45]. Instead, we recommend that designers leverage stickers to add context to the photos and videos people already share.

With regards to presentation style, participants' preferences varied on message format, use case, and personal aesthetic. Although many participants appreciated how analogies helped them make sense of the data, others found them overly wordy or distracting. While some participants found embellished and badge stickers too simple, others appreciated how they could be used to celebrate high amounts or goal achievement. We expect people would prefer platforms support a mix of presentation styles, allowing them to select one which aligns with their situation and preferences. Participants' attitude fatigued from seeing so many Snaps with self-tracked data displayed in similar ways. We expect variety is crucial, as is continuing to make including overlays optional.

\subsection{Future Opportunities}

Prior work has demonstrated the benefit of personalizing analogies for measurements, such as contextualizing a distance for a Chicago-native as "about $5 x$ the distance between you and 
Wrigley Field" [33]. Audience-specific overlays could lead to interpretable and interesting contextualization of data. For example, shared duration analogies could be contextualized as the time it would take to drive or walk to a nearby landmark, with audience members receiving different analogies based on their location. If an audience member collects similar data themselves, analogies could also provide comparisons (e.g., " $1.3 x$ your current heart rate", " $3 x$ more that you've listened to Lizzo this week").

Animation can be a useful way of drawing attention to trends in visualized data [23]. We imagine animated stickers could be a useful tool for demonstrating progress across Snaps shared in a story. For example, embellishments could animate from the previously-shared value to the current value as a person takes additional steps or listens to an artist more times. Animated stickers in direct messages could also drive interest in one-off direct messages, such as a stopwatch ticking up to an amount when sharing duration.

Prior work on sharing of personal informatics data examines messages where the data is the focus of what is being shared (e.g., "Fust wanted to share my heart rate with you, it is currently at 91 bpm" [37], "journaled 45 minutes of elliptical" [45]). Using self-tracked data to supplement the photos people already want to share backgrounds the data slightly. We suspect this approach is still sufficient for the self-presentation and celebration goals people have when sharing tracked data $[25,31,56]$.

Although people tend to add stickers or data-driven filters on ephemeral social media, many archival platforms also include annotation features for images and video. On archival platforms, data-driven stickers could further be used to preserve some history between successive posts, such as the amount of time spent working on a long-term project or the amount of progress towards a weight loss goal. However, prior work suggests that tensions would emerge around whether audiences are too broad to value the accomplishments [19,47]. Future work could experimentally evaluate what platforms people feel self-tracked data is best suited for.

How to support people in authoring data-driven stickers poses important design challenges. People desire the ability to flexibly represent their data [4,22], so we expect people will appreciate tools which enable them to choose from a wide array of available stickers. People may appreciate systems which allow them to choose sticker background colors or fonts which best align with how they want to use the medium to present themselves or best match the other content they are sharing [2]. Systems could also allow people to manipulate what fields data is encoded into, drawing inspiration from prior systems [32]. For example, a sticker could animate its size relative to numeric data, starting and ending small for a low step count or animating form small to large for a high one.

The principles in this paper offer initial guidelines towards integrating stickers driven by personal informatics data into ephemeral platforms. We expect testing these sticker styles with participants on an ephemeral platform would lead to useful findings on what data-sharing goals are well-served by ephemeral social media and what are better-served for other platforms.

\section{CONCLUSION}

We contribute design recommendations for sharing personal informatics data via overlays on ephemeral social media, demonstrating that these overlays are entertaining and informative. The badge, embellished, and analogy stickers we designed can each offer value when explaining moments or celebrating them, though preference depends on circumstance and aesthetics. When possible, the design of overlays should reflect the domain being shared and allow the sharer to use their own photo as a background. Integrating these stickers into ephemeral 
platforms and evaluating them could lead to useful insights about when people find it most useful to include personal informatics data in posts to ephemeral social media.

\section{ACKNOWLEDGMENTS}

We thank Liangze Yu for assistance in developing early drafts of the stickers, the HAI lab for feedback on the survey design, and Elena Agapie and Jessica Schroeder for feedback on the framing and contribution. This work was funded in part by the National Science Foundation under award IIS-1850389 and UCI Council on Research, Computing, and Libraries (CORCL).

\section{REFERENCES}

[1] Saleem Alhabash and Mengyan Ma. (2017). A Tale of Four Platforms: Motivations and Uses of Facebook, Twitter, Instagram, and Snapchat Among College Students? Social Media and Society, 3(1). http://doi.org/gfs4qf

[2] Marina Amâncio. (2017). "Put it in your Story": Digital Storytelling in Instagram and Snapchat Stories.

[3] Fereshteh Amini, Khalad Hasan, Andrea Bunt, and Pourang Irani. (2017). Data representations for in-situ exploration of health and fitness data. Proceedings of the EAI International Conference on Pervasive Computing Technologies for Healthcare (PervasiveHealth 2017), 163-172. http://doi.org/dv9g

[4] Amid Ayobi, Tobias Sonne, Paul Marshall, and Anna L. Cox. (2018). Flexible and Mindful Self-Tracking: Design Implications from Paper Bullet Journals. Proceedings of the SIGCHI Conference on Human Factors in Computing Systems (CHI 2018). http://doi.org/c32p

[5] Elizabeth Bales, Kevin A. Li, and William Griwsold. (2011). CoupleVIBE: Mobile Implicit Communication to Improve Awareness for (Long-Distance) Couples. Proceedings of the ACM Conference on Computer Supported Cooperative Work (CSCW 2011), 65-74. http://doi.org/c8xvm5

[6] Louise Barkhuus, Barry Brown, Marek Bell, Malcolm Hall, Scott Sherwood, and Matthew Chalmers. (2008). From Awareness to Repartee: Sharing Location within Social Groups. Proceeding of the SIGCHI conference on Human factors in computing systems (CHI 2008), 497. http://doi.org/cbhgqd

[7] Scott Bateman, Regan L Mandryk, Carl Gutwin, Aaron Genest, David Mcdine, and Christopher Brooks. (2010). Useful Junk? The Effects of Visual Embellishment on Comprehension and Memorability of Charts. Proceedings of the SIGCHI Conference on Human Factors in Computing Systems (CHI 2010), 2573-2582. http://doi.org/crhdc4

[8] Eric P. S. Baumer, Sherri Jean Katz, Jill E. Freeman, Phil Adams, Amy L. Gonzales, JP Pollak, Daniela Retelny, Jeff Niederdeppe, Christine M. Olson, and Geri K. Gay. (2012). Prescriptive Persuasion and Open-Ended Social Awareness: Expanding the Design Space of Mobile Health. Proceedings of the ACM Conference on Computer Supported Cooperative Work (CSCW 2012), 475-484. http://doi.org/bbkm

[9] Joseph B. Bayer, Nicole B. Ellison, Sarita Y. Schoenebeck, and Emily B. Falk. (2016). Sharing the Small Moments: Ephemeral Social Interaction on Snapchat. Information Communication and Society, 19(7), 956-977. http://doi.org/gghnzv

[10] Frank Bentley, Konrad Tollmar, Peter Stephenson, Laura Levy, Brian Jones, Scott Robertson, Ed Price, Richard Catrambone, and Jeff Wilson. (2013). Health Mashups: Presenting Statistical Patterns between Wellbeing Data and Context in Natural Language to Promote Behavior Change. ACM Transactions on Computer-Human Interaction (TOCHI), 20(5), 1-27. http://doi.org/f23f6c

[11] Michelle A. Borkin, Azalea A. Vo, Zoya Bylinskii, Phillip Isola, Shashank Sunkavalli, Aude Oliva, and Hanspeter Pfister. (2013). What makes a visualization memorable. IEEE Transactions on Visualization and Computer Graphics, 19(12), 2306-2315. http://doi.org/f5h3pd

[12] Monica Caraway, Daniel A. Epstein, and Sean A. Munson. (2017). Friends Don't Need Receipts: The Curious Case of Social Awareness Streams in the Mobile Payment App Venmo. Proceedings of the ACM on Human-Computer Interaction, 1(CSCW), 28:1--28:17. http://doi.org/cqdv

[13] Fanny Chevalier, Romain Vuillemot, and Guia Gali. (2013). Using Concrete Scales: A Practical Framework for Effective Visual Depiction of Complex Measures. IEEE Transactions on Visualizations and Computer Graphics (TVCG), 19(12). http://doi.org/f5h3k4

[14] Chia-Fang Chung, Elena Agapie, Jessica Schroeder, Sonali Mishra, James Fogarty, and Sean A. Munson. (2017). When Personal Tracking Becomes Social: Examining the Use of Instagram for Healthy Eating. Proceedings of the SIGCHI Conference on Human Factors in Computing Systems (CHI 2017), 1674-1687. http://doi.org/ccq4

[15] Sunny Consolvo, Katherine Everitt, Ian Smith, and James A. Landay. (2006). Design Requirements for Technologies that Encourage Physical Activity. Proceedings of the SIGCHI Conference on Human Factors in Computing Systems (CHI 2006), 457. http://doi.org/b2wmz3

[16] Felicia Cordeiro, Daniel A. Epstein, Edison Thomaz, Elizabeth Bales, Arvind K. Jagannathan, Gregory D. Abowd, 
and James Fogarty. (2015). Barriers and Negative Nudges: Exploring Challenges in Food Journaling. Proceedings of the 33rd Annual ACM Conference on Human Factors in Computing Systems - CHI '15, 1159-1162. http://doi.org/bbdt

[17] Franco Curmi, Maria Angela Ferrario, Jen Southern, and Jon Whittle. (2013). HeartLink: Open Broadcast of Live Biometric Data to Social Networks. Proceedings of the SIGCHI Conference on Human Factors in Computing Systems (CHI 2013), 1749-1758. http://doi.org/bjh7

[18] Daniel A. Epstein, Alan Borning, and James Fogarty. (2013). Fine-Grained Sharing of Sensed Physical Activity: A Value Sensitive Approach. Proceedings of the ACM International foint Conference on Pervasive and Ubiquitous Computing (UbiComp 2013), 489-498. http://doi.org/bdsq

[19] Daniel A. Epstein, Bradley H. Jacobson, Elizabeth Bales, David W. McDonald, and Sean A. Munson. (2015). From "nobody cares" to "way to go!": A Design Framework for Social Sharing in Personal Informatics. Proceedings of the ACM Conference on Computer Supported Cooperative Work (CSCW 2015), 1622-1636. http://doi.org/bbks

[20] Daniel A. Epstein, An Ping, James Fogarty, and Sean A. Munson. (2015). A Lived Informatics Model of Personal Informatics. Proceedings of the ACM International Joint Conference on Pervasive and Ubiquitous Computing (UbiComp 2015), 731-742. http://doi.org/bdsr

[21] Daniel A Epstein, Felicia Cordeiro, James Fogarty, Gary Hsieh, and Sean A Munson. (2016). Crumbs: Lightweight Daily Food Challenges to Promote Engagement and Mindfulness. Proceedings of the SIGCHI Conference on Human Factors in Computing Systems (CHI 2016), 5632-5644. http://doi.org/bjh8

[22] Daniel A Epstein, Mira Dontcheva, James Fogarty, and Sean A Munson. (2020). Yarn: Adding Meaning to Shared Personal Data through Structured Storytelling. Proceedings of the Graphics Interface Conference (GI 2020).

[23] Danyel Fisher. (2010). Animation for Visualization: Opportunities and Drawbacks. In Beautiful Visualization. 329352.

[24] Rúben Gouveia, Fábio Pereira, Evangelos Karapanos, Sean A. Munson, and Marc Hassenzahl. (2016). Exploring the Design Space of Glanceable Feedback for Physical Activity Trackers. Proceedings of the ACM International foint Conference on Pervasive and Ubiquitous Computing (UbiComp 2016), 144-155. http://doi.org/dv9h

[25] Shion Guha and Jeremy Birnholtz. (2013). Can You See Me Now? Location, Visibility and the Management of Impressions on foursquare. Proceedings of the International Conference on Human-Computer Interaction with Mobile Devices and Services (MobileHCI 2013), 183-192. http://doi.org/ccwb

[26] Xinning Gui, Yu Chen, Clara Caldeira, Dan Xiao, and Yunan Chen. (2017). When Fitness Meets Social Networks: Investigating Fitness Tracking and Social Practices on WeRun. Proceedings of the ACM Conference on Human Factors in Computing Systems (CHI 2017), 1647-1659. http://doi.org/ccwc

[27] Hana Habib, Neil Shah, and Rajan Vaish. (2019). Impact of Contextual Factors on Snapchat Public Sharing. Proceedings of the SIGCHI Conference on Human Factors in Computing Systems (CHI 2019), 1-13. http://doi.org/dv9j

[28] Brent Hecht, Lichan Hong, Bongwon Suh, and Ed H. Chi. (2011). Tweets from Justin Bieber's Heart: The Dynamics of the "Location" Field in User Profiles. Proceedings of the SIGCHI Conference on Human Factors in Computing Systems (CHI 2011), 237. http://doi.org/bk7dcf

[29] Jessica Hullman, Eytan Adar, and Priti Shah. (2011). Benefitting InfoVis with Visual Difficulties. IEEE Transactions on Visualization and Computer Graphics (TVCG), 17(December), 1-7. http://doi.org/df5c8r

[30] Jessica Hullman, Yea-Seul Kim, Francis Nguyen, Lauren Speers, and Maneesh Agrawala. (2018). Improving Comprehension of Measurements Using Concrete Re-expression Strategies. Proceedings of the SIGCHI Conference on Human Factors in Computing Systems (CHI 2018), 1-12. http://doi.org/dv9k

[31] Kevin O. Hwang, Allison J. Ottenbacher, Angela P. Green, M. Roseann Cannon-Diehl, Oneka Richardson, Elmer V. Bernstam, and Eric J. Thomas. (2010). Social Support in an Internet Weight Loss Community. International fournal of Medical Informatics, 79(1), 5-13. http://doi.org/dtbr7m

[32] Nam Wook Kim, Hyejin Im, Nathalie Henry Riche, Alicia Wang, Krzysztof Gajos, and Hanspeter Pfister. (2019). DataSelfie: Empowering People to Design Personalized Visuals to Represent Their Data. Proceedings of the SIGCHI Conference on Human Factors in Computing Systems (CHI 2019), 1-12. http://doi.org/gf2b82

[33] Yea-Seul Kim, Jessica Hullman, and Maneesh Agrawala. (2016). Generating Personalized Spatial Analogies for Distances and Areas. Proceedings of the SIGCHI Conference on Human Factors in Computing Systems (CHI 2016), 3848. http://doi.org/dv9m

[34] Jieun Lee and Ilyoo B. Hong. (2016). Predicting Positive User Responses to Social Media Advertising: The Roles of Emotional Appeal, Informativeness, and Creativity. International fournal of Information Management, 36(3), 360373. http://doi.org/drdm

[35] Ian Li, Anind Dey, and Jodi Forlizzi. (2010). A Stage-Based Model of Personal Informatics Systems. Proceedings of the SIGCHI Conference on Human Factors in Computing Systems (CHI 2010), 557. http://doi.org/bh8zsb

[36] Janne Lindqvist, Justin Cranshaw, Jason Wiese, Jason Hong, and John Zimmerman. (2011). I'm the Mayor of My House: Examining Why People Use foursquare - a Social-Driven Location Sharing Application. Proceedings of the SIGCHI Conference on Human Factors in Computing Systems (CHI 2011), 2409. http://doi.org/c956b2

[37] Fannie Liu, Laura Dabbish, and Geoff Kaufman. (2017). Supporting Social Interactions with an Expressive Heart

PACM on Human-Computer Interaction, Vol. 4, No. CSCW2, Article 95, Publication date: October 2020. 
Rate Sharing Application. Proceedings of the ACM on Interactive, Mobile, Wearable and Ubiquitous Technologies, 1(3), 1-26. http://doi.org/cc62

[38] Fannie Liu, Mario Esparza, Maria Pavlovskaia, Geoff Kaufman, Laura Dabbish, and Andrés Monroy-Hernández. (2019). Animo: Sharing Biosignals on a Smartwatch for Lightweight Social Connection. Proceedings of the ACM on Interactive, Mobile, Wearable and Ubiquitous Technologies, 3(1), 1-19. http://doi.org/dv9n

[39] Kai Lukoff, Taoxi Li, Yuan Zhuang, and Brian Y. Lim. (2018). TableChat: Mobile Food Journaling to Facilitate Family Support for Healthy Eating. Proceedings of the ACM on Human-Computer Interaction, 2(CSCW), 1-28. http://doi.org/dv9p

[40] Diane Maloney-Krichmar and Jenny Preece. (2005). A Multilevel Analysis of Sociability, Usability, and Community Dynamics in an Online Health Community. ACM Transactions on Computer-Human Interaction (TOCHI), 12(2), 201-232. http://doi.org/fm3r9t

[41] Sarah McRoberts, Haiwei Ma, Andrew Hall, and Svetlana Yarosh. (2017). Share First, Save Later: Performance of Self through Snapchat Stories. Proceedings of the SIGCHI Conference on Human Factors in Computing Systems (CHI 2017). http://doi.org/ccwf

[42] Measure of Things. http://www.bluebulbprojects.com/MeasureOfThings/default.php

[43] Andrew Vande Moere, Helen Purchase, and K. U. Leuven. (2011). On the role of design in information visualization. Information Visualization, 10(4), 356-371. http://doi.org/djn5hr

[44] Meredith Ringel Morris, Jaime Teevan, and Katrina Panovich. (2010). What do people ask their social networks, and why? Proceedings of the SIGCHI Conference on Human Factors in Computing Systems (CHI 2010), 1739. http://doi.org/c69

[45] Sean A. Munson and Sunny Consolvo. (2012). Exploring Goal-setting, Rewards, Self-monitoring, and Sharing to Motivate Physical Activity. Proceedings of the International Conference on Pervasive Computing Technologies for Healthcare (PervasiveHealth 2012), 25-32. http://doi.org/bbn8

[46] Sean A. Munson, Erin Krupka, Caroline Richardson, and Paul Resnick. (2015). Effects of Public Commitments and Accountability in a Technology-Supported Physical Activity Intervention. Proceedings of the SIGCHI Conference on Human Factors in Computing Systems (CHI 2015), 1135-1144. http://doi.org/bbkw

[47] Mark W. Newman, Debra Lauterbach, Sean A. Munson, Paul Resnick, and Margaret E. Morris. (2011). "It's Not That I Don't Have Problems, i'm Just Not Putting Them on Facebook": Challenges and Opportunities in Using Online Social Networks for Health. Proceedings of the ACM Conference on Computer Supported Cooperative Work (CSCW 2011), 341. http://doi.org/bcvgg7

[48] John Allen Paulos. (2001). Innumeracy: Mathematical Illiteracy and its Consequences. Holt McDougal.

[49] Andrew Perrin and Monica Anderson. (2019). Share of U.S. Adults using Social Media, Including Facebook, is Mostly Unchanged Since 2018. https://www.pewresearch.org/fact-tank/2019/04/10/share-of-u-s-adults-using-social-mediaincluding-facebook-is-mostly-unchanged-since-2018/

[50] Lukasz Piwek and Adam Joinson. (2016). "What do They Snapchat About?" Patterns of use in Time-Limited Instant Messaging Service. Computers in Human Behavior, 54, 358-367. http://doi.org/gfzzmz

[51] Zachary Pousman, John T. Stasko, and Michael Mateas. (2007). Casual Information Visualization: Depictions of Data in Everyday Life. IEEE Transactions on Visualization and Computer Graphics (TVCG), 13(6), 1145-1152. http://doi.org/ffd536

[52] Edward Segel and Jeffrey Heer. (2010). Narrative visualization: Telling Stories with Data. IEEE Transactions on Visualization and Computer Graphics (TVCG), 16(6), 1139-1148. http://doi.org/dnqx5m

[53] Suvi Silfverberg, Lassi A Liikkanen, Airi Lampinen, and South Hall. (2011). "I'll press Play, but I won't listen": Profile Work in a Music-focused Social Network Service. Proceedings of the ACM Conference on Computer Supported Collaborative Work (CSCW 2011), 207-216. http://doi.org/c6xqvd

[54] Meredith M. Skeels, Kenton T. Unruh, Christopher Powell, and Wanda Pratt. (2010). Catalyzing Social Support for Breast Cancer Patients. Proceedings of the SIGCHI Conference on Human Factors in Computing Systems (CHI 2010), 173-182. http://doi.org/cnxsm9

[55] (2018). State of Social Video. https://www.vidmob.com/resource/us-state-of-social-video-2018/

[56] Rannie Teodoro and Mor Naaman. (2013). Fitter with Twitter: Understanding Personal Health and Fitness Activity in Social Media. Proceedings of the International AAAI Conference on Weblogs and Social Media (ICWSM 2013), 611620.

[57] Tammy Toscos, Anne Faber, Kay Connelly, and Adity Mutsuddi Upoma. (2008). Encouraging Physical Activity in Teens: Can Technology Help Reduce Barriers to Physical Activity in Adolescent Girls? Proceedings of the International Conference on Pervasive Computing Technologies for Healthcare (PervasiveHealth 2008), $218-221$. http://doi.org/cvmffx

[58] Edward R. Tufte. (1983). The Visual Display of Quantitative Information. Graphics Press.

[59] Suvi Uski and Airi Lampinen. (2016). Social Norms and Self-Presentation on Social Network Sites: Profile Work in Action. New Media and Society, 18(3), 447-464. http://doi.org/dv9q 
[60] J. Mitchell Vaterlaus, Kathryn Barnett, Cesia Roche, and Jimmy A. Young. (2016). "Snapchat is More Personal”: An Exploratory Study on Snapchat Behaviors and Young Adult Interpersonal Relationships. Computers in Human Behavior, 62, 594-601. http://doi.org/gghnz2

[61] Lea Verou. CSS3 Patterns Gallery. https://leaverou.github.io/css3patterns/

[62] We Are Social, Hootsuite, and DataReportal. Distribution of Snapchat users worldwide as of July 2019, by age and gender. https://www.statista.com/statistics/933948/snapchat-global-user-age-distribution/

[63] Jason Wiese, Sauvik Das, Jason I. Hong, and John Zimmerman. (2017). Evolving the Ecosystem of Personal Behavioral Data. Human-Computer Interaction, 32(5-6), 447-510. http://doi.org/ccwh

[64] Wolfram|Alpha. https://www.wolframalpha.com/

[65] Paweł W. Woźniak, Anton Fedosov, Eleonora Mencarini, and Kristina Knaving. (2017). Soil, Rock and Snow: On Designing for Information Sharing in Outdoor Sports. Proceedings of the 2017 ACM Conference on Designing Interactive Systems (DIS 2017), 611-623. http://doi.org/dv9r

[66] Bin Xu, Pamara Chang, Christopher L. Welker, Natalya N. Bazarova, and Dan Cosley. (2016). Automatic Archiving versus Default Deletion: What Snapchat Tells Us About Ephemerality in Design. Proceedings of the ACM Conference on Computer Supported Collaborative Work (CSCW 2016), 1660-1673. http://doi.org/ggdt2p

[67] Heng Xu, Xin Luo, John M. Carroll, and Mary Beth Rosson. (2011). The Personalization Privacy Paradox: An Exploratory Study of Decision Making Process for Location-Aware Marketing. Decision Support Systems, 51(1), 4252. http://doi.org/d3w5ch

[68] Heng Xu, Lih Bin Oh, and Hock Hai Teo. (2009). Perceived effectiveness of text vs. multimedia Location-Based Advertising messaging. International fournal of Mobile Communications, 7(2), 154. http://doi.org/dk8v2m

[69] Xuan Zhao, Niloufar Salehi, Sasha Naranjit, Sara Alwaalan, Stephen Voida, and Dan Cosley. (2013). The Many Faces of Facebook: Experiencing Social Media as Performance, Exhibition, and Personal Archive. Proceedings of the SIGCHI Conference on Human Factors in Computing Systems (CHI 2013), 1-10. http://doi.org/bjjp

Submitted January 2020, revised June 2020, accepted July 2020. 